This item was submitted to Loughborough's Research Repository by the author.

Items in Figshare are protected by copyright, with all rights reserved, unless otherwise indicated.

\title{
Differential measurements using two Laser Rotational Vibrometers: dynamic
} backlash

PLEASE CITE THE PUBLISHED VERSION

http://dx.doi.org/10.1117/12.693072

PUBLISHER

(C) Society of Photo-Optical Instrumentation Engineers (SPIE)

VERSION

VoR (Version of Record)

LICENCE

CC BY-NC-ND 4.0

REPOSITORY RECORD

Martin, Peter, and Steve Rothberg. 2019. "Differential Measurements Using Two Laser Rotational Vibrometers: Dynamic Backlash". figshare. https://hdl.handle.net/2134/19726. 


\title{
Differential measurements using two Laser Rotational Vibrometers: dynamic backlash
}

\author{
P Martin, S.J Rothberg \\ Wolfson School of Mechanical \& Manufacturing Engineering, \\ Loughborough University, \\ Loughborough. Leicestershire. LE11 3TU. England. UK. \\ Tel: (44) 1509227519 \\ Email: p.martin@lboro.ac.uk
}

\begin{abstract}
The Laser Rotational Vibrometer is well suited to non-contact measurement of angular vibration on rotating targets, particularly by virtue of inherent insensitivity to target shape and translational motion. A differential measurement is proposed using two Vibrometers to allow a calculation of dynamic backlash between spur gears from relative tangential displacements. It is known that target motions, such as rotation, produce changes in the speckle pattern on the photodetector which add noise to the Vibrometer output. The significance of noise is always increased when differential measurements are required. The noise produced by the speckle pattern is apparent but the data reveals that it is possible to make the dynamic backlash measurement in this way. The dynamic backlash measurement is verified against equivalent high speed video footage, confirming the suitability of Laser Vibrometry in this application.
\end{abstract}

Keywords: Laser Rotational Vibrometer, Vibrometry, Differential Measurement, Dynamic Backlash, Speckle Noise

\section{INTRODUCTION}

Dynamic backlash measurement can be beneficial in the assessment of gear noise, tooth wear and gear life. In this paper, a differential measurement using two laser rotational vibrometers is proposed as a means to measure dynamic backlash. Dynamic backlash is calculated using the relative tangential displacement of the gears at the respective pitch circle radii. A high speed, digital video of the mesh point of the gears is also recorded for the purposes of validation.

\subsection{Laser Rotational Vibrometer}

The Laser Rotational Vibrometer is well suited to non-contact measurement of angular vibration on rotating targets. Parallel laser beams $(<1 \mathrm{~mW})$ are incident on the rotating target, aligned perpendicular to the axis of rotation for maximum sensitivity. The parallel beam configuration provides insensitivities to target shape and whole body translational motions. Optical elements collect a proportion of the Doppler-shifted, backscattered light and the instrument output is proportional to the instantaneous angular velocity of the illuminated cross-section of the target.

\subsection{Laser speckle}

When coherent light is scattered from an optically rough surface a speckle pattern is formed ${ }^{1}$. The surface height deviations cause the component wavelets to become de-phased. In the far-field of the scatter, constructive and destructive interference of the de-phased but still coherent component wavelets produces a pattern of random light and dark regions known as a speckle pattern. Target motions, such as rotation, produce changes in the speckle pattern and these changes contribute noise to the signal output ${ }^{2}$. This speckle noise is generally pseudo-random in nature, exhibiting approximately equal amplitude peaks at a fundamental frequency equal to the rotational frequency and at harmonics up to very high order ${ }^{3}$. This so-called pseudo-vibration ${ }^{2}$ is, in general, low-level but significant in that it is indistinguishable 
from genuine vibrations and particularly prominent on measurements from rotating targets. Momentary loss of signal can also be attributed to speckle effects. The uncorrelated nature of the speckle noise in each individual measurement causes speckle noise to be even more significant in a differential measurement ${ }^{4}$.

\section{DIFFERENTIAL MEASUREMENT: DYNAMIC BACKLASH}

Backlash is necessary within a meshing gear set for many reasons amongst which are to aid lubrication, to allow for variations in tooth profiles and to accommodate build tolerances. For a set of meshing gears, dynamic backlash can be defined as the time dependent separation between meshing teeth. With the assumption that the gears remain rigid, this differential measurement uses the relative tangential displacement to quantify the backlash at the mesh point. The relative tangential displacement, $s(t)$, can be shown to be

$$
s(t)=r_{A} \theta_{A}(0)-r_{B} \theta_{B}(0)+\int_{0}^{t} r_{A} \Omega_{A}-r_{B} \Omega_{B} d t
$$

and

$$
s(t)=\left(r_{A} \theta_{A}(0)-r_{B} \theta_{B}(0)\right)+\left(r_{A} \bar{\Omega}_{A}-r_{B} \bar{\Omega}_{B}\right) t+\left(r_{A} \int_{0}^{t} \Delta \Omega_{A}(t) d t-r_{B} \int_{0}^{t} \Delta \Omega_{B}(t) d t\right)
$$

where subscripts $A$ and $B$ denote relation to gears $\mathrm{A}$ and $\mathrm{B}$ respectively, $r_{A}$ and $r_{B}$ are the contact radii, $\theta_{A}(0)$ and $\theta_{B}(0)$ are the initial angular positions, $\Omega_{A}=\bar{\Omega}_{A}+\Delta \Omega_{A}$ and $\Omega_{B}=\bar{\Omega}_{B}+\Delta \Omega_{B}, \Omega_{\mathrm{A}}$ and $\Omega_{\mathrm{B}}$ are the total angular velocities, $\bar{\Omega}_{A}$ and $\bar{\Omega}_{B}$ are the mean angular velocities and $\Delta \Omega_{A}(t)$ and $\Delta \Omega_{B}(t)$ are the alternating component of angular velocities. The first bracketed term is the initial relative position. This cannot be extracted from the Vibrometer data and, unless acquisition is taken from a known relative position which in general is impractical, this quantity will remain unknown. The second bracketed term is the mean component of the relative tangential displacement and is zero for meshing gears. The third term is the alternating component of the relative tangential displacement and is defined here as the dynamic backlash.

The backlash in the gears is governed by the contact radius for each gear. It is entirely possible that the separation distance between centres of rotation may not be the sum of the pitch circle radii, in which case contact will not occur at the pitch circle radii. The contact radii are dictated by the number of gear teeth, the distance between the centres of rotation and the pitch circle radii but the tangential velocities of each gear are always equal at the contact position when there is contact and the ratio of mean angular velocities is always uniquely set by the ratio of the number teeth. Therefore, the following relationships apply

$$
\frac{N_{A}}{N_{B}}=\frac{\bar{\Omega}_{B}}{\bar{\Omega}_{A}}=\frac{r_{A}}{r_{B}}=\frac{r_{A P C R}}{r_{B P C R}}=\frac{\Delta r_{A}}{\Delta r_{B}}
$$

where $N_{A}$ and $N_{B}$ are the number of teeth on each gear, $r_{A P C R}$ and $r_{B P C R}$ are the pitch circle radii, and $\Delta r_{A}$ and $\Delta r_{B}$ are the distances from pitch circle radii to the point of tooth contact along a line between the axes of rotation of the gears. The pitch circle radii are known quantities and are convenient as a means for comparison between gear sets. However any relative tangential position can be measured as long as the radial positions conform to the ratio as seen in equation (3). Therefore, dynamic backlash is further defined in this paper as the time dependent tangential separation at the pitch circle radii using the third term in equation (2).

Using this term and knowledge of the gear set geometry, it is possible to calculate the relative tangential displacement and hence the dynamic backlash, $B(t)$. Using Simpson's rule,

$$
B(t)=\frac{1}{6 f_{s}} \sum_{p=3}^{P}\left[\kappa_{A} r_{A P C D}\left(V_{A}(p)+4 V_{A}(p-1)+V_{A}(p-2)\right)-\kappa_{B} r_{B P C D}\left(V_{B}(p)+4 V_{B}(p-1)+V_{B}(p-2)\right)\right]
$$

where $\Delta \Omega_{A}(t)=\kappa_{A} V_{A}(t), \Delta \Omega_{B}(t)=\kappa_{B} V_{B}(t), f_{s}$ is the acquisition sample rate, $P$ is the total number of samples, $p$ is the sample index and $V_{A}(t)$ and $V_{B}(t)$ are the time dependent voltage outputs of the Vibrometers measuring from gears A 
and $\mathrm{B}$ respectively. The time, $t$, at which the calculation is performed is given as $t=P / f_{s}$. The respective Vibrometer output sensitivities, $\kappa_{\mathrm{A}}$ and $\kappa_{\mathrm{B}}$, are set by the experimental geometrical configuration.

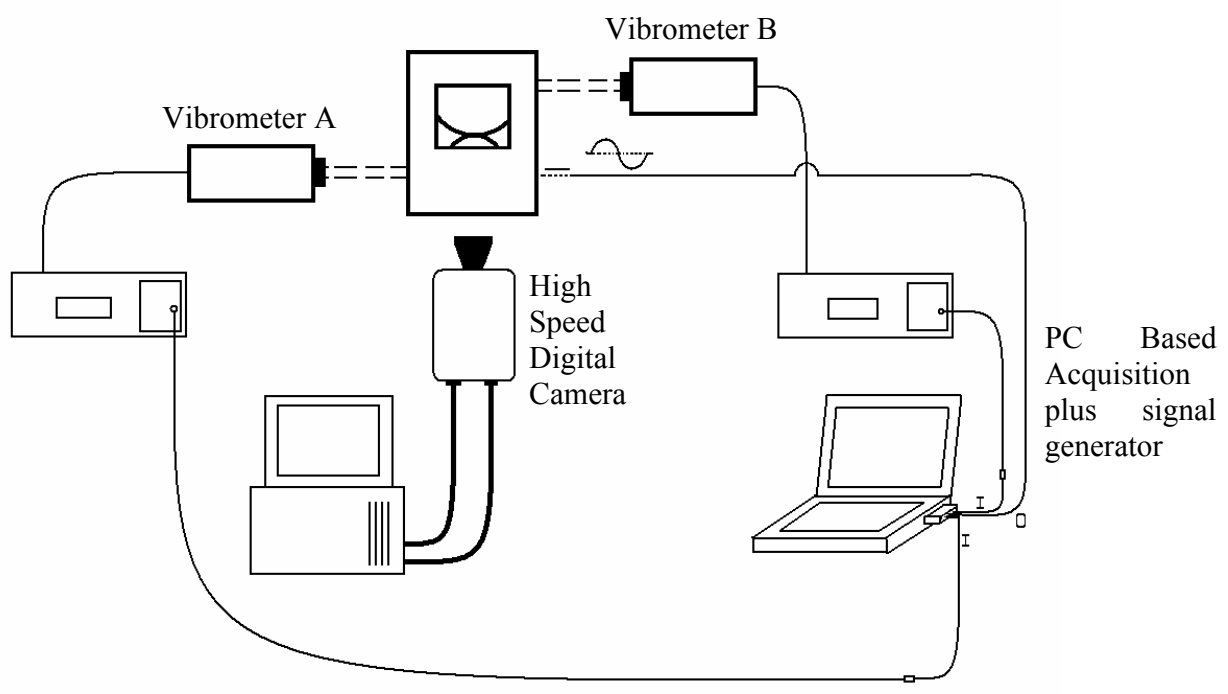

Figure 1a - Experimental set-up of dynamic backlash measurement

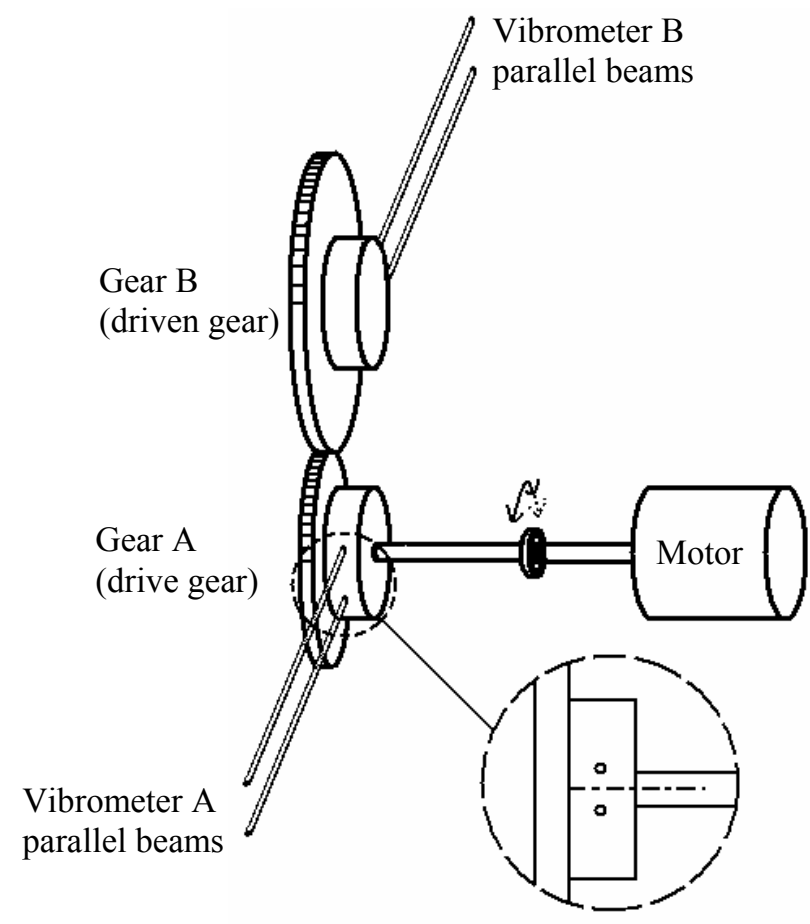

Figure $1 \mathrm{~b}$ - Laser Rotational Vibrometer incident on gear bosses treated with retro-reflective tape 


\section{EXPERIMENT}

\subsection{Experimental set-up}

Figure 1a shows the experimental setup. Two Rotational Vibrometers measure oscillations in rotational velocity on a meshing pair of rotating spur gears. The two pairs of beams were incident on the bosses of the respective gears and aligned parallel with each other and perpendicular to each rotational axis as shown in Figure 1b. The gears had Pitch Circle Diameters (PCD) of $72 \mathrm{~mm}$ and $40 \mathrm{~mm}$ with 90 teeth and 50 teeth respectively, with a circular pitch of $2.513 \mathrm{~mm}$. Drive was via direct coupling of a 12V DC motor to the 40PCD gear with the 72PCD gear unloaded. Each gear boss was treated with retro-reflective tape. The meshing point of the gears was white light illuminated with a halogen lamp and imaged with a high speed digital camera which had a frame rate of $4.5 \mathrm{kHz}$ and a spatial resolution of approximately $28 \mu \mathrm{m} / \mathrm{pixel}$. The motor was driven with a DC voltage on top of which a nominally sinusoidal oscillation can be induced. The variation in motor drive voltage promotes a fluctuation in rotational speed of the drive gear and sets up a dynamic backlash.

\subsection{Image processing}

Verification of the dynamic backlash is made by correlating data calculated from the Vibrometers with measurements taken from video images. Verification is made at the pitch circle radii, but with knowledge of the axial separation it is possible to calculate the relative tangential displacement at the radial contact position. The video footage from the high speed camera is dissected into its individual frames. Pixel measurements of the circular pitch are made and a spatial resolution calculated. Pixel measurements are taken from between teeth at the pitch circle radii and converted into a backlash measurement. A backlash range can be found by measurements taken from subsequent tooth face impacts.

Figure 2 shows an example of the method by which the measurements are taken to calculate the backlash range. A vertical broken line shows the position of the pitch circle radius of the bottom drive gear and a vertical solid line shows the position of the pitch circle radius of the top driven gear. In frame 00180 at the pitch circle radii there is a tangential distance of 11 pixels which equates to a backlash measurement of $-0.307 \mathrm{~mm}$, in frame 00218 the backlash measurement is $0.195 \mathrm{~mm}$, therefore the dynamic backlash range equates to $0.5026 \mathrm{~mm}$.

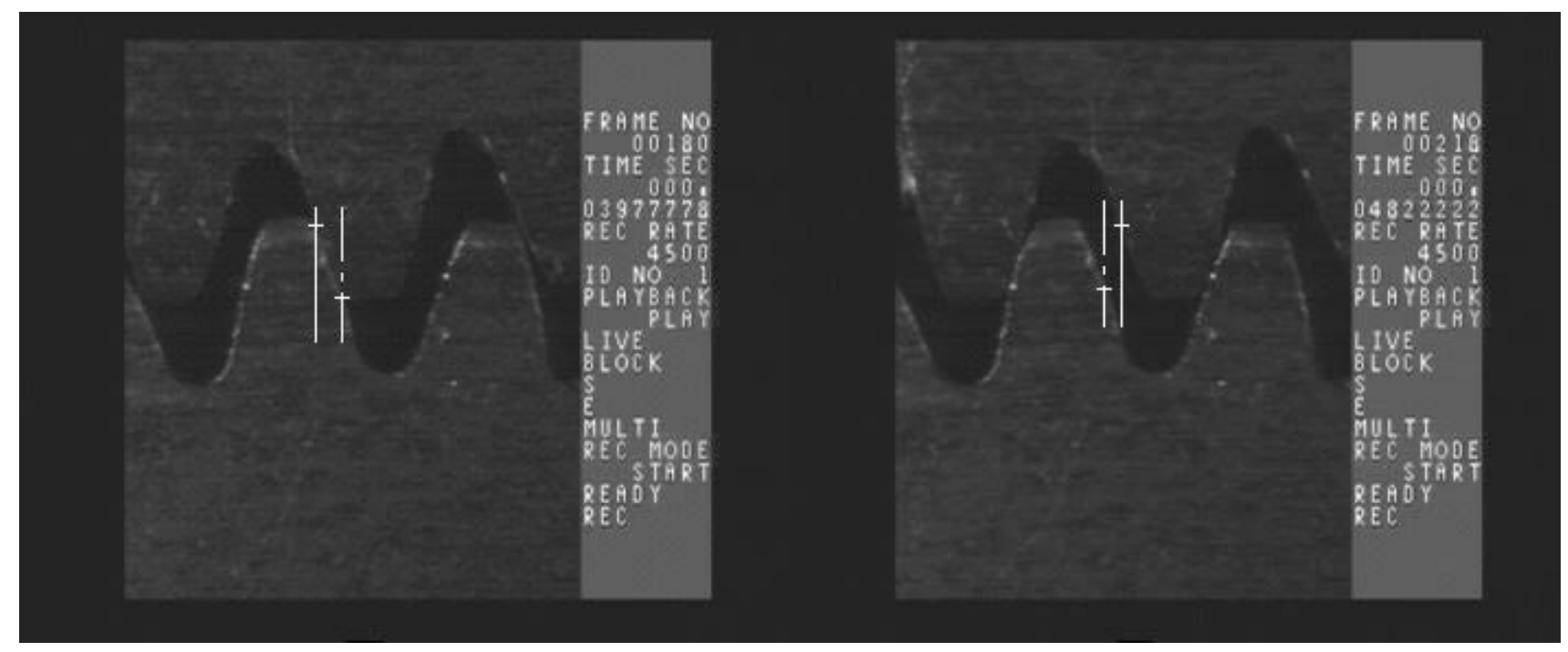

Figure 2 - Backlash measurement on consecutive tooth face impacts. 


\section{RESULTS \& DISCUSSION}

\subsection{Laser Vibrometer verification with high speed video footage}

Experiments have been conducted with sinusoidal rotational excitation of the drive gear, with and without whole body rotation. With whole body rotation and sinusoidal oscillation, the gear undergoes a speed fluctuation about the mean rotational speed. With sinusoidal excitation, only, the gear oscillates angularly about a mean position.

Figure 3 is an extract from video footage of a $60 \mathrm{~Hz}$ sinusoidal oscillation of the drive gear in the absence of whole body rotation. The individual frames show the meshing process with 13 frame intervals. Imaged is the mesh point of a drive gear (bottom) and driven gear (top). For oscillating targets with or without whole body rotation, the motion of the gears can be described as follows:

- Frame 1: Teeth in contact, clockwise rotation of the drive gear and anti-clockwise rotation of the driven gear.

- Frames 2\&3: After reaching the extreme of its oscillation cycle, the drive gear begins to rotate anti-clockwise, contact is lost and the driven gear continues to rotate anti-clockwise.

- Frame 4: Drive gear continues to rotate anti-clockwise until impact occurs between teeth. This rapidly decelerates the driven gear and causes it to rotate clockwise.

- Frames 5\&6: After reaching the extreme of its oscillation cycle, the drive gear begins to rotate clockwise again, contact is lost and the driven gear continues to rotate clockwise.

With whole body rotation, the behaviour of the gears is identical, the oscillations are simply superimposed on top of the mean rotations.

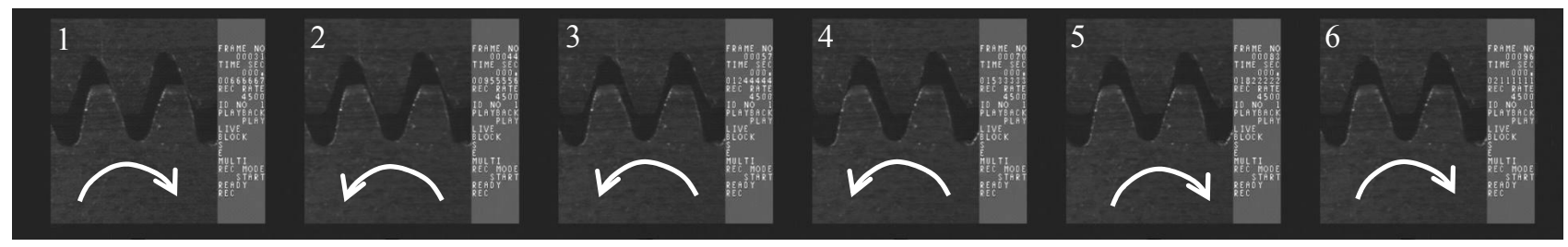

Figure 3 -Video footage showing gear motion with $60 \mathrm{~Hz}$ sinusoidal excitation (no whole body rotation). Bottom gear is the drive gear and top gear is the driven gear.

For the drive gear rotating at $217 \mathrm{rpm}$ (rotation speed is limited to approximately 280rpm when using the high speed video) with a $60 \mathrm{~Hz}$ sinusoidal excitation, Figure 4 shows the dynamic backlash as calculated from equation (4). Figure 5 displays the dynamic backlash at the pitch circle radii for a gear set with $60 \mathrm{~Hz}$ sinusoidal oscillation but without whole body rotation. The dynamic backlash ranges and duration of events are consistent between Figure 4 and Figure 5 . This serves to confirm that this method can reliably evaluate backlash under rotating conditions. A periodic waveform of fundamental frequency $60 \mathrm{~Hz}$ is observed and this is attributed to the sinusoidal modulation of the drive. Interpretation of the waveform is consistent with the process described in Figure 3. The sections of virtually zero slope at (approximate) maxima and minima are the periods of tooth contact, on average of $2.3 \mathrm{~ms}$ duration. This duration is consistent with the $2.4 \pm 0.2 \mathrm{~ms}$ estimated from the relevant frames of the video footage. Within these regions of tooth contact are small oscillations. The magnitude of these oscillations is below $8 \mu \mathrm{m}$ which is below the resolution limit of the camera. These oscillations are likely to be a bounce ${ }^{5}$. The sections with a large change in dynamic backlash equate to periods where the driven gear is not in contact with the drive gear and this is the transition stage from one tooth face to another. The average duration of this transition is estimated to be $5.9 \mathrm{~ms}$ which is consistent with $5.5 \pm 0.2 \mathrm{~ms}$ estimated from the video footage. The dynamic backlash range on average is $0.51 \mathrm{~mm}$ which, as described in Section 3.2 , is consistent with that found from the video footage.

This limited rotational speed is not representative of typical industrial applications so experiments were conducted to observe any issues associated with a measurement at increased rotational speed. Figure 6 shows calculated backlash when the drive gear is rotating at $1800 \mathrm{rpm}$ with a periodic but harmonically distorted oscillation with a $50 \mathrm{~Hz}$ 
fundamental frequency superimposed on the drive. This oscillation is a prominent feature of the waveform containing regions of tooth contact and also periods of tooth separation. In this experiment, a reduced axial separation of the gear centres has resulted in a slightly decreased peak dynamic backlash.

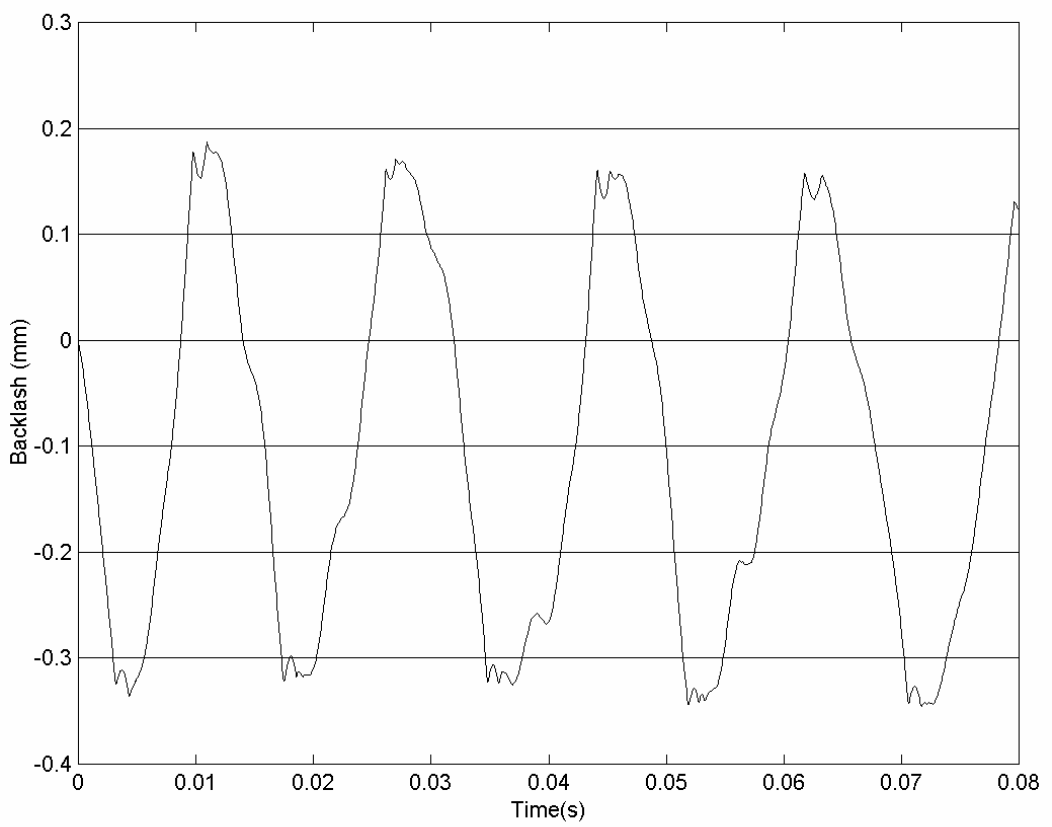

Figure 4 - Backlash with sinusoidal excitation at $60 \mathrm{~Hz}$, with whole body rotation of the drive gear at $217 \mathrm{rpm}$

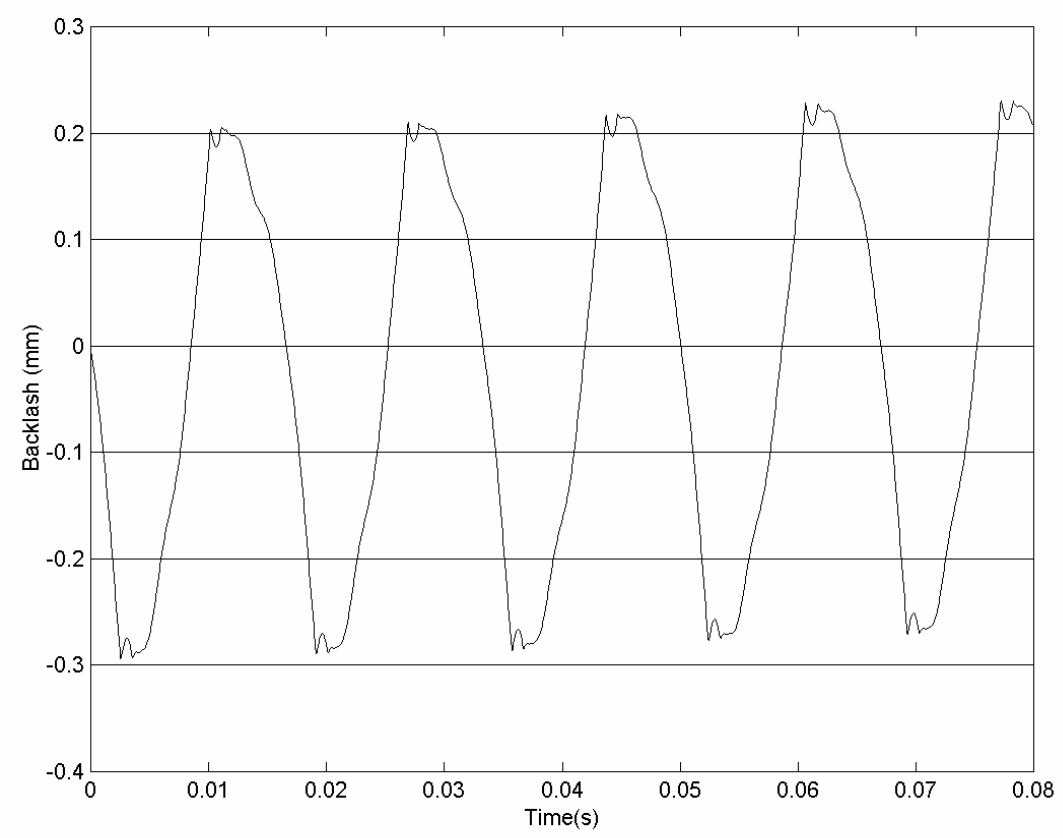

Figure 5 - Backlash with sinusoidal excitation at $60 \mathrm{~Hz}$, without whole body rotation 


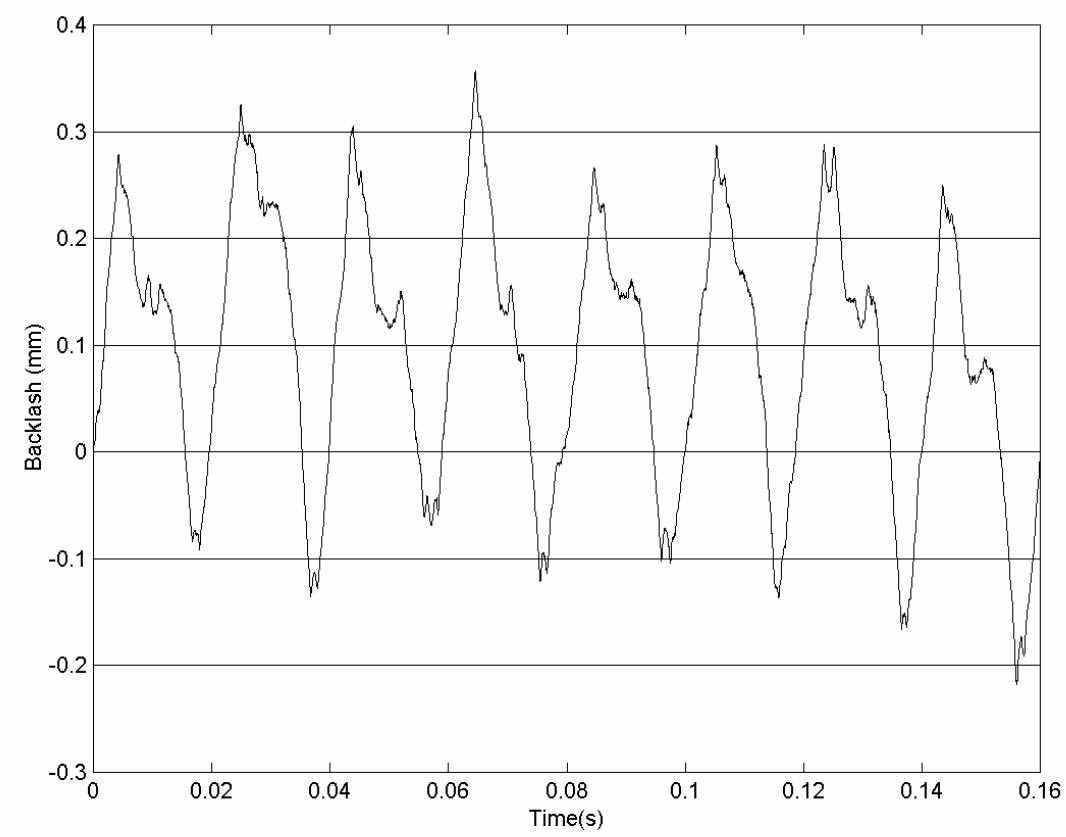

Figure 6 - Backlash, drive gear rotating at 1800rpm and oscillating with $50 \mathrm{~Hz}$ fundamental frequency.

\subsection{Effect of noise on dynamic backlash calculation}

The effect that noise, particularly speckle noise, has on the dynamic backlash measurement technique has been examined through simulation. The gear set motion has been simplified to create two waveforms to represent the voltage outputs from the Vibrometers as shown in Figure 7a.
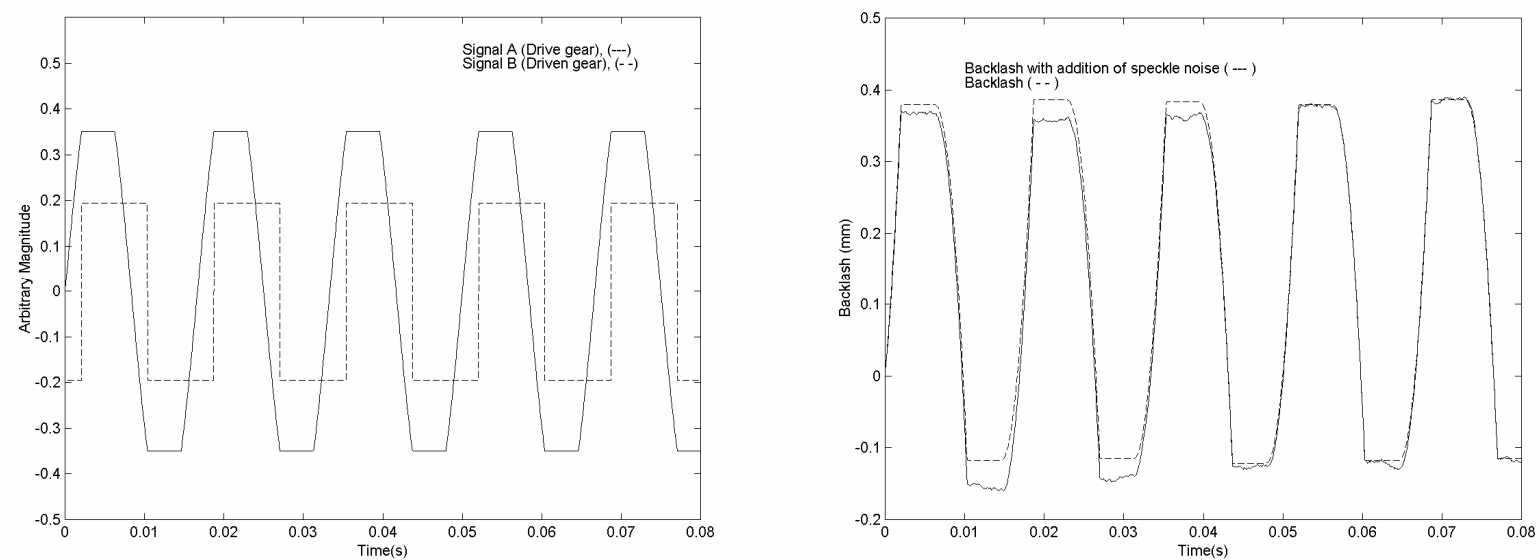

Figure 7 -a) Simulated Vibrometer outputs before addition of noise.

b) Calculated dynamic backlash with and without the addition of speckle noise.

The simulation is intended to mimic the experimental configuration which produced the data in Figure 4 . The waveforms have been appropriately scaled for the difference in radii and produce a backlash comparable with that seen in Figure 4. In the simulation, the drive gear oscillates sinusoidally and the driven gear remains at a constant velocity during periods 
without tooth contacts. The drive gear sinusoidal motion has been clipped to make the angular velocity constant during periods of contact. The duration of contact is made equivalent (15\% of period) to that observed in real Vibrometer data and video footage. To simulate speckle noise, pseudo-random signals are created by a normally distributed random number generator. The signals repeat at the same frequency as rotation frequency and are scaled in accordance with the noise seen on the real Vibrometer data. It can be seen in Figure $7 \mathrm{~b}$ how the addition of speckle noise causes a low frequency drift of the backlash measurement. This results from integration of the lower frequency components of the simulated noise and it is also apparent in Figure 4 and Figure 5.

Figure $8 \mathrm{a}$ is the spectrum of this simulated backlash, while Figure $8 \mathrm{~b}$ is the spectrum of the same simulated backlash but with the addition of speckle noise. Figure 8 a shows the dc term associated with the lack of a known starting position as well as peaks at the fundamental frequency of $60 \mathrm{~Hz}$ and, at diminishing levels, the $3^{\text {rd }}$ and $5^{\text {th }}$ harmonics. These peaks are characteristic of a square-wave, to which the calculated backlash can be approximated. The increased low frequency components responsible for the drift observed in the simulated backlash calculations including speckle noise are readily apparent in Figure $8 \mathrm{~b}$ but the peaks associated with backlash are easily identifiable and in agreement with those in Figure 8 a.
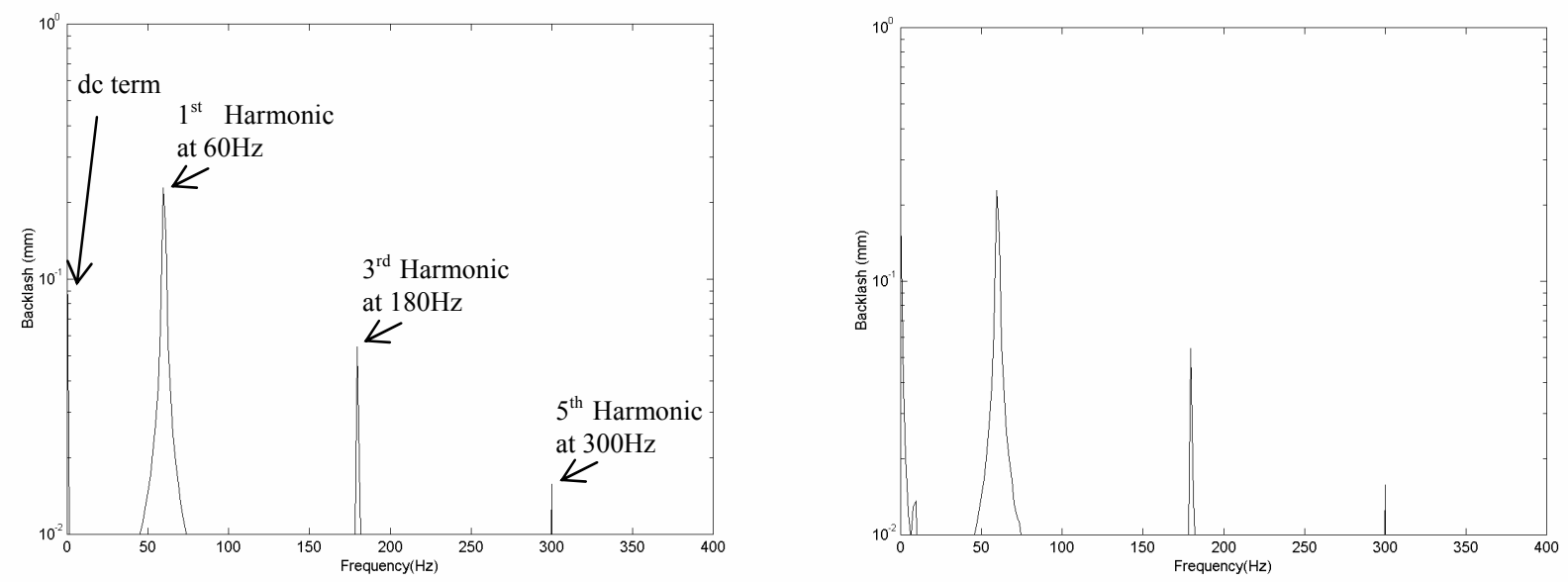

Figure 8 -a) Spectrum of simulated backlash with $60 \mathrm{~Hz}$ oscillation,

b) Spectrum of simulated backlash with $60 \mathrm{~Hz}$ oscillation and addition of noise from speckle

\section{CONCLUSION}

This paper has demonstrated that it is possible to measure dynamic backlash between gears using two Laser Rotational Vibrometers. Procedures for experimental data acquisition and subsequent analysis have been developed and verified through use of a high speed video camera and through simulation. Use of the camera constrained rotation of the gears to low speeds so further experiments at elevated speeds have been conducted successfully using only the Vibrometers. Although speckle noise is present in the individual measurements, it does not prevent a dynamic backlash measurement from being made even though the method involves differential measurements. Experimental data exhibited a noticeable low frequency drift. Simulation has shown how low frequency noise components, such as those that might originate from speckle noise, cause the drift in the calculated backlash that is so apparent in the time domain but observation in the frequency domain shows how the signal components associated with the dynamic backlash are unaffected.

\section{ACKNOWLEDGEMENTS}

The authors would like to acknowledge the Engineering and Physical Sciences Research Council (EPSRC) for financial support and Perkins Engines for providing the inspiration for the project. 


\section{REFERENCES}

1 Dainty, J.C., Laser Speckle and Related Phenomena, J.C. Dainty, Editor. 1984, Springer-Verlag. p. 1-7.

2 S.J. Rothberg, J. R. Baker \& N. A. Halliwell, Laser Vibrometery: Pseudo-Vibrations, Journal of Sound and Vibration, 1989, 135(3). P516-522

3 S. J. Rothberg \& B. Halkon, Laser vibrometry meets laser speckle, Proc of SPIE Sixth International conference on Vibration Measurements by Laser Techniques: Advances and Applications, 22-25 June 2004, Vol 5503. p280-291

4 B Halkon, \& S. J. Rothberg, Automatic post-processing of laser vibrometry data for rotor vibration measurements, Eighth International Conference on Vibrations in Rotating Machinery. P215-230

5. J. Stein \& C. Wang, Automatic Detection of Clearance in Mechanical Systems: Experimental Validation, Mechanical Systems and Signal Processing, 1996 10(4), 395-412 